\title{
Suppression of Tunneling of Superconducting Vortices Caused by a Remote Gate: Example of an Extended Object Tunneling
}

\author{
K. Michaeli and A. M. Finkel'stein \\ Department of Condensed Matter Physics, The Weizmann Institute of Science, Rehovot 76100, Israel
}

\begin{abstract}
We discuss a recent experiment in which the resistance of a superconducting film has been measured in magnetic field. A strong decrease of the superconducting film resistance has been observed when a metallic gate is placed above the film. We study how the magnetic coupling between vortices in a thin superconducting film and electrons in a remote unbiased gate suppresses the tunneling rate of the vortices. We examine two general approaches to analyze tunneling in the presence of slow low-energy degrees of freedom: the functional-integral and scattering formalisms. In the first one, the response of the electrons inside the metallic gate to a change in the vortex position is described by the "tunneling with dissipation". We consider the Eddy current induced in the gate by the magnetic flux of the vortex as a result of tunneling. In the second approach, the response is given in terms of scattering of the electrons by the magnetic flux of the vortex in a way similar to the Aharonov-Bohm scattering. A sudden change in the vortex position leads to the Orthogonality Catastrophe that opposes the vortex tunneling. We show that the magnetic coupling between the vortices and the electrons inside the gate can lead to a dramatic suppression of the vortex tunneling, restoring the superconducting property in accord with the experiment.
\end{abstract}

\section{INTRODUCTION}

The vortex motion in superconductors is a source of energy losses, destroying the perfect conductivity of the superconductors. The dissipation is caused by non-superconducting electrons located inside the vortex core $\underline{\underline{1}}$ The pinning potential created by impurities opposes the motion of vortices. This potential results from the action of the impurities on the vortex core averaged over the area of the core. Therefore, the pinning potential has minima typically separated by a distance of order of the coherence length $\xi \stackrel{2,3}{2}$ Vortices may change their positions either by thermal activation ${ }^{4}$ or by quantum tunneling between the potential minima at low enough temperatures,$\frac{5,6,7,8}{1}$ In this context, the observation of a strong decrease of the resistance when an unbiased metallic gate is placed above an amorphous superconducting film ${ }^{9}$ is of great interest. We believe that this experiment provides a strong argument that at low temperature the motion of vortices is indeed realized by quantum tunneling (in the experiment described in Ref. 9 it occurs at $T \lesssim 0.1 K)$. If so, the tunneling of a vortex in a thin superconducting film is a unique example of tunneling of a very extended "object".

In the discussed experiment, the resistance of a superconducting film has been measured at various magnetic fields, both with and without a gate. In the absence of a gate, in magnetic fields lower than the critical one $\left(H<H_{C 2}\right)$, the resistance initially decreases with lowering the temperature, but eventually saturates at a finite value. The saturation indicates the possibility of vortex tunneling. When an unbiased metallic gate is placed above the superconducting film the resistance reduces significantly with no indication of saturating at a finite resistance when $T \rightarrow 0$. Remarkably, the effect of the gate becomes noticeable at the same temperatures where the resistance of the ungated film starts to satu- rate. It is worth mentioning that the gate is separated from the film by an oxide layer of $160 \AA$. Therefore, the film is thermally isolated from the gate ruling out the possibility that the saturation of the resistance in the ungated film can be attributed to heating. In this paper we identify the mechanism causing the suppression of the vortex motion in the presence of the gate, which is effective in the tunneling regime only. The fact that the finite resistance at low temperatures has been eliminated by placing a remote isolated gate strongly confirms the tunneling nature of the vortex motion.

In the experiment of Ref. 9 the film thickness is $a \approx$ $30 \AA$ and the gate thickness is $d \approx 400 \AA$. An important feature indicating that the gate and the film are well separated, is that the superconducting transition temperature, $T_{c}$, as well as the critical magnetic field, are practically unchanged by adding the gate. Since the gate does not affect the superconductivity at $T \approx T_{c}$ when it is the weakest, its influence on the superconducting properties, like the energy gap, at lower temperatures can be ignored. One should also keep in mind that the gate does not influence the resistance of the film when the vortex motion is thermally activated. In view of all of the above, we concentrate only on examining the influence of the gate on the vortex tunneling rate. We assumed that the superconducting film and the gate are magnetically coupled via the magnetic field of the vortices that pierces through the gate.

We employ here the following strategy. We accept the tunneling of vortices at low temperatures as an established experimental fact. We do not try to calculate the tunneling rate of the vortices. Instead, we concentrate on how the response of the electrons inside the gate to a change of the vortex position suppresses the tunneling rate. With this question in mind, in the complex problem of the vortex tunneling, we wish to isolate the effect induced by the gate.

In fact, little is known about the motion of vortices at 


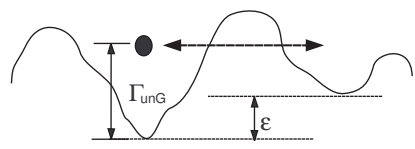

(a)



(b)
FIG. 1: The vortex in the effective potential landscape is represented by a hopping "particle". (a) The tunneling rate in the absence of the gate $\Gamma_{\text {un }}$ exceeds the energy difference between the potential minima $\varepsilon$; the vortices are in a "metallic" phase (b) The tunneling rate is reduced by the gate to $\Gamma_{G}<\varepsilon$, and the system of vortices becomes an "insulator".

low temperatures $\underline{10}$ Fortunately, for studying the role of the gate, it is sufficient to assume that the change in the vortex position is a discrete tunneling event. This can be a tunneling of a single vortex, a bundle of vortices, or topological defects such as dislocation pairs in the case of a vortex lattice (or a glass state). Phenomenologically, the change in the vortex position can be described by the hopping Hamiltonian

$$
H=\sum_{i} \varepsilon_{i} a_{i}^{+} a_{i}+\sum_{\langle i, j\rangle}\left(\Gamma_{i j} a_{i}^{+} a_{j}+\text { h.c. }\right) .
$$

According to the standard criterion of the MetalInsulator transition 11 , the ratio of the variation of the potential minima $\varepsilon=\left\langle\varepsilon_{i}\right\rangle$ to the typical value of the tunneling rates $\Gamma=\left\langle\Gamma_{i j}\right\rangle$ specifies whether the vortices are itinerant or localized. The finite resistance at low temperature in the absence of the gate indicates that the vortices are mobile, i.e., the tunneling rate in the ungated film $\Gamma_{u n G}>\varepsilon$. The dissipationless nature of the superconducting film is revived when the tunneling rate is reduced by the gate to $\Gamma_{G}<\varepsilon$; see Fig. 1 which illustrates the two cases. Thus, we interpret the experiment $\underline{9}$ as a transition from "metallic" to "insulating" phases in a system of tunneling vortices that is induced by the gate. (Because of the strong interaction between the vortices, it may be enough to have a fraction of vortices with a suppressed probability of tunneling acting as the pinning centers for the entire ensemble of vortices.)

The fact that the tunneling of the vortices can be blocked by placing a gate above the film indicates that the tunneling event gives rise to a dramatic response of the electrons inside the gate. This response can be analyzed in terms of low-energy electron-hole excitations "decorating" the tunneling event. One may consider the cloud of virtual excitations as part of the tunneling process that lasts long after the change in the vortex positions occurs. In the present work we concentrate on the response of the electrons to the tunneling that can lead to the strong suppression of the tunneling rate.

One may better understand the specifics of tunneling with the participation of low-energy degrees of freedom, i.e., in the presence of dissipative environment, using the picture given by Iordanskii and one of the authors $\underline{\underline{12}}$ Originally it described the quantum formation of a nu- cleation center in the decay of a metastable macroscopic state, but it could also provide a general perspective. The quantum nucleation is an example of a tunneling process in which a large number of degrees of freedom participate. Alternatively, this kind of process can be treated as a tunneling of an artificial "particle" in a multidimensional space. When low-energy degrees of freedom are involved in the process of quantum nucleation, the nucleation develops in two stages $\frac{12}{=}$ Namely, the motion of the "particle" in this multi-dimensional space along the trajectory minimizing the imaginary time action consists of fast and slow stages.

The slow stage appears because of the long time needed for the slow low-energy degrees of freedom to adjust themselves to the new state of the fast degrees of freedom $\underline{\underline{13}}$ This time is much longer than needed for fast the degrees of freedom to complete the tunneling. That is why the tunneling has to develop in two stages. It has been shown in Ref. 12 that despite the fact that the low-energy degrees of freedom yield only a small contribution to the energy of the barrier, their participation in the tunneling process increases parametrically the overall tunneling time. This results in a large increase of the action and, correspondingly, in the strong suppression of the tunneling rate $\underline{\underline{14}}$

The described picture of changing a quantum state in the presence of low-energy degrees of freedom is rather typical for condensed matter systems. In the course of the fast stage of the process a quantum mechanical object changes its state (position, spin projection, phase of the Josephson junction, etc). The accompanying slow degrees of freedom act as an environment for the fast degrees of freedom. In the discussed problem of tunneling in the gated superconducting film, the tunneling of the vortex from one potential minimum to another corresponds to the fast stage. During the slow stage, the electrons inside the gate adjust their state to the new position of the vortex.

The ensemble of electron-hole pairs in the gate represents the low-energy degrees of freedom of the environment. The environment produces the most significant effect on tunneling at large time differences when the slow degrees of freedom have enough time to develop. Naturally, the effect is the strongest when the tunneling occurs between states that are almost degenerate. In the latter case the long time response can considerably reduce or even block the tunneling 15,16 (this statement is often formulated in terms of the dissipative Quantum Phase Transition 17 ). With this in mind, we concentrate only on the slow stage that develops when the tunneling of the vortex degrees of freedom is mostly accomplished without specifying how the fast stage develops.

In this paper, we examine two different approaches to describe the response of the electrons inside the gate to the change of the vortex position. In Secs. III and III we consider the dissipative Eddy (Foucault) currents induced in the gate which continue long after the vortex changed its position. We formulated the effect of the 
gate on the vortex tunneling in terms of the effective action of a vortex. We integrate out the environmental degrees of freedom inside the gate, and obtain the dissipative term in the effective action. This allows us to consider the vortex tunneling in the gated superconducting film in the context of the well-known problem of tunneling in the presence of a dissipative environment $\underline{18}$, Sec. IV] Alternatively, one can analyze the slow stage in terms of the scattering of electrons. In Sec. $\mathrm{V}$ we describe the elastic scattering of the gate electrons on the vector potential of the magnetic field of the vortex. The zero overlap between the states of the electrons before and after the change of the scattering potential is known generally as the Orthogonality Catastrophe $\frac{19}{}(\mathrm{OC})$. We show that the OC caused by the change in the vortex position, effectively suppresses the vortex tunneling. The novel element here $\mathrm{e}^{20}$ is that the $O C$ is connected to the Aharonov-Bohm effect 21.22 We obtained that the $O C$ is significantly more effective in suppressing the tunneling rate than the Eddy current. In Sec. VD we discuss the relation between the two approaches and the peculiarities of the tunneling of an extended object, such as a vortex in a thin superconducting film.

\section{EDDY CURRENTS INSIDE THE GATE}

The magnetic field of the vortex inside a superconducting film is similar to the magnetic field of a solenoid with a radius equal to the magnetic penetration depth $\lambda$ which can be very large $\stackrel{23}{\underline{n}}$ Outside of the film, the magnetic field decays as a function of the height and deflects into the radial direction 24,25 :

$$
\mathbf{A}_{v o r}(\mathbf{r}, z ; \mathbf{R})=\frac{\alpha \Phi_{0}}{\lambda} \int \frac{d^{2} q}{(2 \pi)^{2}} e^{i \mathbf{q r}-q|z|} \frac{i \mathbf{q} \times \hat{z}}{q^{2}\left(\lambda^{-1}+2 q\right)} .
$$

Here $\mathbf{r}$ is the radial vector in cylindrical coordinates with the origin at the vortex center $\mathbf{R}$. Since a vortex in a film can move only in the $x-y$ plane, $\mathbf{R}$ is a two dimensional vector. The parameter $\alpha=1 / 2$ is the total flux of a vortex in a superconductor measured in units $\Phi_{0}=2 \pi \hbar c / e$.

In the case of a thin superconducting film, a Pearl vortex is a macroscopically large object. The penetration depth $\lambda$ is ${ }^{23}$ :

$$
\lambda=\lambda_{3 D}^{2} / a \quad \lambda \gg a,
$$

where $a$ is the thicknesses of the superconducting film, $\lambda_{3 D}$ is the penetration depth in a disordered bulk superconductor. In Eq. (3), $\lambda_{3 D} \sim \lambda_{L}\left(\xi_{0} / \ell\right)^{1 / 2}$, where $\lambda_{L}=\left(m c^{2} / 4 \pi n e^{2}\right)^{1 / 2}$ and $\xi_{0}$ are the London length and the coherence length of a clean superconductor; $\ell$ is the mean free path.

In the experiment of Ref. 9 the gate thickness $d$ is much smaller than $\lambda$. Because of the exponential decay of the Fourier components in the $z$-direction, see Eq. (2), momenta that contribute mostly are limited to $q \lesssim 1 / d$.

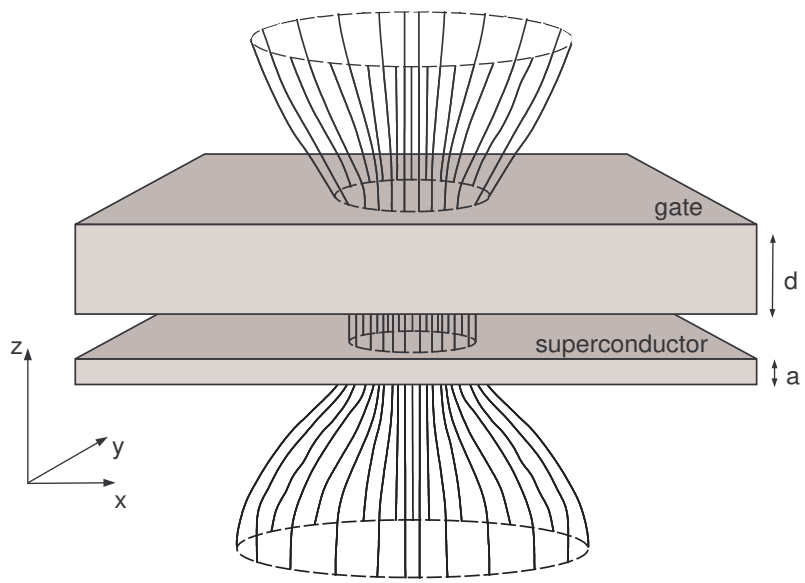

FIG. 2: Superconducting film magnetically coupled to a metallic gate. The magnetic field of the vortex pierces through the gate. In the experiment of Ref. $9 d \approx 400 \AA, a \approx 30 \AA$, and the insulating layer between the gate and the film is $160 \AA$ thick.

More accurately, $q \lesssim \min \left\{d^{-1}, \delta r^{-1}\right\}$, where $\delta r$ is the typical distance that a vortex has to tunnel, $\delta r \sim \xi$. To avoid unnecessary complications, we ignore the space between the superconducting film and the gate since it is considerably smaller than the gate thickness, see Fig. 2. Then, for small momenta that we are interested in, the deflection of the magnetic field in the space between the film and the gate can be neglected.

To describe the tunneling of a vortex in the presence of a gate one has to deal with an imaginary time action:

$$
S=S_{s c}+S_{\text {gate }}+S_{\text {int }} .
$$

In what follows we discuss each term in the action $S$.

The term $S_{s c}$ is an action of the superconducting film in the absence of the gate. Since we are not trying to solve the problem of the vortex tunneling in full scale, but are interested only in the effect of the gate on the tunneling rate, this part of the action is not specified.

In writing $S_{\text {gate }}$ which describes the dynamics of the electrons in the gate one should keep in mind the following argument. The charge and current densities relevant for the long tail response of electrons inside the gate to the tunneling of the vortex are characterized by large length and time scales. Therefore, their dynamics can be described macroscopically. Since the deviations of the charge and current densities from their equilibrium values are small, the action that describes the dynamics of their fluctuations should be consistent with the FluctuationDissipation Theorem (FDT). (Examples of such an approach can be found in the calculation of the dephasing time of the cooperons due to the electromagnetic fluctuations ${ }^{26}$, and also in a macroscopic calculation of the zero bias anomaly $: 27$ )

The current in the gate has two contributions. One is the Ohmic response to the electric field, while the other one is the diffusive current from the gradients of the den- 
sity; $\mathbf{J}(\mathbf{r}, \tau)=\mathbf{J}_{\text {ohmic }}(\mathbf{r}, \tau)-D \nabla \rho(\mathbf{r}, \tau)$. The fluctuations of the charge and current densities can be expressed through the correlation function of the Ohmic part of the current written in terms of the Matsubara frequency as follows:

$$
\begin{gathered}
\hat{K}_{i, j}^{-1}\left(\mathbf{k}, i \omega_{n}\right)=\left\langle\mathbf{J}_{o h m i c}^{i}\left(\mathbf{k}, i \omega_{n}\right) \mathbf{J}_{o h m i c}^{j}\left(-\mathbf{k},-i \omega_{n}\right)\right\rangle \\
\quad=\sigma_{i, j}\left(\mathbf{k}, \omega_{n}\right)\left|\omega_{n}\right|+\sigma_{i, i^{\prime}}\left(\mathbf{k}, \omega_{n}\right) D_{j, j^{\prime}}\left(\mathbf{k}, \omega_{n}\right) k_{i^{\prime}} k_{j^{\prime}} .
\end{gathered}
$$

Here the diffusion constants tensor $\hat{D}$ and the conductivity $\hat{\sigma}$ are connected through the Einstein's relation $\hat{\sigma}=e^{2}(d n / d \mu) \hat{D}$. The gate, being a simple homogeneous metal, is adequately described by the Drude formula. We assume that the external magnetic field is classically weak, $\omega_{c} \tau \ll 1$, so that we can ignore the Hall conductivity in the gate; $\tau$ is the mean free time in the gate. Since we are interested in the low frequency and long wave length behavior only, we take the conductivity $\sigma$ to be constant.

Following the above arguments, one can write the gate action $S_{\text {gate }}$ for the charge and current densities inside the gate in the Matsubara time:

$$
\begin{aligned}
S_{\text {gate }} & =\frac{1}{2} \int_{0}^{\beta} d \tau_{1} d \tau_{2} \int d \mathbf{r}_{1} d \mathbf{r}_{2} L_{F D T}\left[\mathbf{r}_{1}, \tau_{1} ; \mathbf{r}_{2}, \tau_{2}\right] \\
& +\int_{0}^{\beta} d \tau \int d \mathbf{r}\left[\mathcal{L}_{\text {cont }}+\mathcal{L}_{\text {Max }}\right]
\end{aligned}
$$

The first term in the above action describes the charge and current density fluctuations in accordance with the FDT:

$$
\begin{gathered}
L_{F D T}=\left[\mathbf{J}\left(\mathbf{r}_{1}, \tau_{1}\right)+\hat{D} \nabla \rho\left(\mathbf{r}_{1}, \tau_{1}\right)\right] \hat{K}\left(\mathbf{r}_{1}-\mathbf{r}_{2}, \tau_{1}-\tau_{2}\right) \\
{\left[\mathbf{J}\left(\mathbf{r}_{2}, \tau_{2}\right)+\hat{D} \nabla \rho\left(\mathbf{r}_{2}, \tau_{2}\right)\right] .}
\end{gathered}
$$

Since the fluctuations of the current and density are not independent, the second term in Eq. (6) imposes the charge continuity condition with the use of the Lagrange multiplier $\phi$ :

$$
\mathcal{L}_{\text {cont }}=\phi(\mathbf{r}, \tau)(i \dot{\rho}(\mathbf{r}, \tau)+\nabla \mathbf{J}(\mathbf{r}, \tau))
$$

The term $\mathcal{L}_{\text {Maxwell }}$ describes the interactions of the electromagnetic fields and the charge and current densities in the gate in a way that reproduces the Maxwell equations:

$$
\begin{aligned}
\mathcal{L}_{\text {Max }} & =i \frac{1}{c}\left[\mathbf{J} \mathbf{A}_{\text {ind }}+\rho \varphi_{\text {ind }}\right] \\
& +\frac{1}{8 \pi}\left[\left(-\nabla \varphi_{i n d}-\frac{i}{c} \dot{\mathbf{A}}_{\text {ind }}\right)^{2}+\left(\boldsymbol{\nabla} \times \mathbf{A}_{\text {ind }}\right)^{2}\right] .
\end{aligned}
$$

The factor $i$ in the coupling of the electromagnetic fields with the charge and current densities appears because of the imaginary time. 28 The plus in the magnetic field term (which is a consequence of the $i$-factor) is needed to get the repulsive sign in the Ampere interaction of currents, like in the Coulomb interaction of charges. As usually, the total potentials are the sum of the external and induced potentials; in the discussed system the external source is the field of the vortices.

The last term in the action (4), $S_{\text {int }}$, describes the connection between the superconducting film and the gate. The current and charge densities in the gate interact with the vector and scalar potentials created by the superconducting film:

$$
\begin{aligned}
S_{i n t}=i \int_{0}^{\beta} d \tau \int d \mathbf{r}\left\{\frac{1}{c} \mathbf{J}(\mathbf{r},\right. & \tau) \cdot \mathbf{A}_{s c}(\mathbf{r}, \tau) \\
& \left.+\rho(\mathbf{r}, \tau) \varphi_{s c}(\mathbf{r}, \tau)\right\}
\end{aligned}
$$

We are interested in the limited problem of the long tail response of electrons inside the gate that develops when the tunneling of the vortex degrees of freedom is mostly accomplished. Therefore, we consider large enough $\tau$ when one can assume that the deformation of the field $\mathbf{A}_{s c}(\mathbf{r}, \tau)$ appearing during the process of vortex tunneling has been already relaxed. [By deformation we mean the deviation of $\mathbf{A}_{s c}(\mathbf{r}, \tau)$ from the field of the "rigid" vortex centered at $\mathbf{R}(\tau)$, as it is given by Eq. (2). Here $\mathbf{R}(\tau)$ denotes the position of the vortex at time $\tau$.] With this in mind, we put in $S_{\text {int }}$

$$
\mathbf{A}_{s c}(\mathbf{r}, \tau)=\mathbf{A}_{v o r}(\mathbf{r} ; \mathbf{R}(\tau)) \text {. }
$$

Furthermore, we ignore the scalar potential, $\varphi_{s c}(\mathbf{r}, \tau)=$ $\varphi_{\text {vor }}(\mathbf{r} ; \mathbf{R}(\tau))=0$, relying on the known fact that the redistribution of the charge density around the vortex core is negligible. We still have to justify our treatment of the electromagnetic field $\mathbf{A}_{s c}$ as a given external field in the analysis of the long tail response of the gate electrons. We will see in the next section that the magnetic field created by the low-frequency components of the dissipative Eddy currents is much smaller than the field of the vortex, and can perturb the superconducting film only weakly. Therefore, the gate does not provide a substantial feedback effect to the superconducting film during the slow stage.

To get the response of the environment on the vortex motion, one has to integrate out the gate degrees of freedom. Since $S_{\text {gate }}$ and $S_{\text {int }}$ are quadratic in the charge and current densities, this immediately results in

$$
S_{e n v}=\frac{i}{2} \int_{0}^{\beta} d \tau \int d r \frac{1}{c} \mathbf{J}_{c l}(\mathbf{r}, \tau) \cdot \mathbf{A}_{v o r}(\mathbf{r}, \mathbf{R}(\tau)) .
$$

The current $\mathbf{J}_{c l}(\mathbf{r}, \tau)$ has to be found by solving the classical equations of motion. Since the current $\mathbf{J}(\mathbf{r}, \tau)$ describes the long-time response of the electrons in the gate, the term $S_{e n v}$ is non-local in time.

\section{THE SOLUTION OF THE EQUATION OF MOTION}

As we stated above, the main contribution to the induced current emerges from the components of the field 
with $q \lesssim 1 / d$ for which the depletion of the magnetic field of the vortex from the $z$ direction is negligible. Therefore, we can ignore the current component in the $z$-direction and consider only the components that are parallel to the plane. With this in mind, we decompose the current parallel to the plane of the gate into two components: a longitudinal component along the two dimensional vector $q$ and a transverse component perpendicular to it, $\mathbf{J}(\mathbf{q}, \tau)=J^{\|}(\mathbf{q}, \tau) \hat{\mathbf{q}}+J^{\perp}(\mathbf{q}, \tau) \hat{z} \times \hat{\mathbf{q}}$. In the following we use the Fourier transformation for the in-plane coordinates only and keep the vertical coordinate separately. Then, the kernel in Eq. (5) is diagonal in the chosen basis. As it follows from Eq. (21), the vector potential $\mathbf{A}_{\text {vor }}(\mathbf{r})$ contains the transverse component only. Therefore, since the use of the FDT for describing the slow stage implies a linear response to the external field, the longitudinal component $J^{\|}$cannot be generated. This is a direct consequence of the magnetic coupling between the superconducting film and the gate. It is very different from the zero bias anomaly in which only a longitudinal current is generated. $\underline{27}$

The variation of $S_{\text {gate }}+S_{\text {int }}$ with respect to the current and charge densities determines the equations of motion. We use these equations for finding the transverse current:

$$
\begin{aligned}
& \int d z^{\prime} K^{\perp}\left(\mathbf{q} ; z, z^{\prime} ; i \omega_{n}\right) J^{\perp}\left(\mathbf{q}, z^{\prime}, i \omega_{n}\right) \\
& =-\frac{1}{c^{2}} \int d z^{\prime} U\left(\mathbf{q}, z-z^{\prime}\right) J^{\perp}\left(\mathbf{q}, z^{\prime}, i \omega_{n}\right)-i \frac{1}{c} A_{v o r}^{\perp}\left(\mathbf{q}, i \omega_{n}\right) .
\end{aligned}
$$

The L.H.S of this equation is the total vector potential in the transverse direction calculated via its Ohmic response: $J^{\perp}=\left(\sigma \omega_{n} / c\right) A_{\text {total }}^{\perp}$. The first term in the R.H.S is the vector potential of the induced field and the second one is the external potential of the vortex. Neglecting relativistic effects, the instant kernel $U$ can be written as the Fourier transform of the instant in time Biot-Savart kernel $U\left(\mathbf{r}-\mathbf{r}^{\prime}\right)=1 /\left|\mathbf{r}-\mathbf{r}^{\prime}\right|$ with respect to the in-plane momenta, $U\left(\mathbf{q}, z-z^{\prime}\right)=2 \pi e^{-q\left|z-z^{\prime}\right|} / q$.

The above equation for the transverse current can be rewritten in the form:

$$
\int d z^{\prime} L^{\perp}\left(\mathbf{q} ; z, z^{\prime} ; i \omega_{n}\right) J^{\perp}\left(\mathbf{q}, i \omega_{n}\right)=-i \frac{\sigma\left|\omega_{n}\right|}{c} A_{v o r}^{\perp}\left(\mathbf{q}, i \omega_{n}\right),
$$

where the kernel $L^{\perp}$ is

$$
\begin{aligned}
& L^{\perp}\left(\mathbf{q} ; z, z^{\prime} ; i \omega_{n}\right)= \\
& \left\{\begin{array}{r}
\delta\left(z-z^{\prime}\right)+\frac{2 \pi \sigma\left|\omega_{n}\right|}{c^{2}} e^{-q\left|z-z^{\prime}\right|} / q 0 \leq z, z^{\prime} \leq d ; \\
\text { otherwise } .
\end{array}\right.
\end{aligned}
$$

To find the transverse current one has to invert this kernel.

In analogy to the skin-effect, one can define a screening length $\delta\left(\mathbf{q}, \omega_{n}\right)=1 / \sqrt{q^{2}+4 \pi \sigma\left|\omega_{n}\right| / c^{2}}$. [Usually, the skin-effect is discussed in the case of an electromagnetic wave propagating normally to a surface of a metallic slab. The geometry of the problem studied here is different as the magnetic field is normal to the slab surface, while the propagation is parallel to it. Still, the surface current appearing in the gate screens out the high-frequency components of the field in the bulk of the slab.] The kernel $L^{\perp}$ can be inverted in the two limits: (i) for the components of the electromagnetic field that are transparent for the gate, $d / \delta\left(\mathbf{q}, \omega_{n}\right) \ll 1$; or (ii) for the components with $d / \delta\left(\mathbf{q}, \omega_{n}\right) \gg 1$ that are well screened by the surface currents. These are the thin and thick gate limits, respectively.

In the thin gate limit, the change in the current along the $z$ direction is minor, and a reasonable approximation (up to liner terms in $q d$ ) is to consider the current to be homogenous in the $z$ direction, $I_{2 D}=d J^{\perp}\left(\mathbf{q}, z, i \omega_{n}\right)$. Then,

$$
I_{2 D}=-i \frac{\sigma^{2 D}}{1+\frac{2 \pi \sigma^{2 D}\left|\omega_{n}\right|}{q c^{2}}} \frac{\left|\omega_{n}\right|}{c} A_{v o r}^{\perp}\left(\mathbf{q}, i \omega_{n}\right)+O(q d) .
$$

One can observe that the expression for the current $I_{2 D}$ is identical to the current in a two-dimensional system with $\sigma^{2 D}=\sigma d$, and where $2 \pi \sigma^{2 D}\left|\omega_{n}\right| / q c^{2}$ is the current screening operator. [The term "current screening operator" is used here in analogy with the polarization operator. It describes the screening of the transverse component of the time dependent vector potential by the induced currents.]

At low frequencies such that $2 \pi \sigma^{2 D}\left|\omega_{n}\right| / c^{2}<q \lesssim d^{-1}$ (this automatically implies the thin gate limit), the current in the gate screens weakly the field produced by the vortex. The total field is approximately just the field of the vortex, and the current is merely the Ohmic response to it:

$$
I_{2 D}=-i \frac{\sigma^{2 D}\left|\omega_{n}\right|}{c} A_{v o r}^{\perp}\left(\mathbf{q}, i \omega_{n}\right) .
$$

For higher frequencies, $2 \pi \sigma^{2 D}\left|\omega_{n}\right| / c^{2}>q$, but still in the thin gate limit, the situation is rather different. Since the effect of dissipation is stronger for better conducting gates, we are interested in the case when $2 \pi \sigma^{2 D} / c \gg 1$. Then, there is a window $q c^{2} / 2 \pi \sigma^{2 D}<\left|\omega_{n}\right|<q c$ in which the current $I_{2 D}$ obeys a London-like equation ${ }^{24}, I_{2 D}=$ $-i \frac{q c}{2 \pi} A_{v o r}^{\perp}\left(\mathbf{q}, i \omega_{n}\right)$. Since the electrons instantly respond to the potential, the contribution to the action in this limit is not of a dissipative character. Rather, it provides a local in time term acting as an additional potential that should be added to $S_{s c}$. This kind of contribution is not considered here.

For a thick gate, $\delta\left(\mathbf{q}, \omega_{n}\right) \ll d$, the limit of low frequencies does not exist for $q \lesssim d^{-1}$. In this limit the screening length is equal to $\delta\left(\omega_{n}\right)=c / \sqrt{4 \pi \sigma\left|\omega_{n}\right|}$. At such high frequencies the current flows in the reduced volume (which effectively is a thin slab of width $\delta\left(\omega_{n}\right)$ ) as follows:

$$
\begin{aligned}
J^{\perp}\left(\mathbf{q}, z, i \omega_{n}\right) & =-i \frac{c q}{2 \pi} \delta^{-1}\left(\mathbf{q}, \omega_{n}\right) e^{-z / \delta\left(\mathbf{q}, \omega_{n}\right)+q z} \\
& \times A_{\text {vor }}^{\perp}\left(\mathbf{q}, z, i \omega_{n}\right)+O\left(e^{-d / \delta}\right) .
\end{aligned}
$$


Notice that the Fourier components $A_{v o r}^{\perp}(\mathbf{q}, z)$ decay exponentially on $z$ as $e^{-q z}$. Therefore, the factor $e^{q z}$ in the solution above is canceled out leaving the induced surface current with the $z$-dependence $e^{-z / \delta\left(q, \omega_{n}\right)}$. Integrating Eq. (18) in the $z$-direction yields the London-like current $I_{2 D}=-i \frac{q c}{2 \pi} A_{v o r}^{\perp}\left(\mathbf{q}, i \omega_{n}\right)$ exactly as in the thin gate limit.

So far, we have ignored relativistic effects that appear at high frequencies when $\left|\omega_{n}\right| / c>q$. In this case, one has to substitute $q$ by the relativistic combination $\sqrt{q^{2}+\left(\omega_{n} / c\right)^{2}}$. Using the relativistic equations of motion, one can show that the current $\mathbf{J}_{c l}$ is like in the Ohmic regime but with $\sigma^{2 D}$ replaced by $\sigma^{2 D} /\left(1+2 \pi \sigma^{2 D} / c\right)$. In the limit $2 \pi \sigma^{2 D} / c \gg 1$, the dissipation caused by the Cherenkov's radiation 29 corresponds to the effective conductivity equal to $c / 2 \pi$. Still, the effect of the relativistic region is negligible in comparison to the low-frequency contribution to $S_{\text {env }}$.

To conclude, let us come back to our assumption about the absence of a feedback from the gate to the superconducting film. As we have showed above, the most significant contribution to $S_{\text {env }}$ originates from the region $2 \pi\left|\omega_{n}\right| \sigma^{2 D} /\left(q c^{2}\right) \ll 1$ when the Eddy current is in the Ohmic regime. In this limit, the low-frequency components of the vortex field are poorly screened by the induced current in the gate. Hence, the feedback from the gate to the superconducting film can be neglected.

We are ready to obtain the dissipative term in the action describing the effective response of the environmental degrees of freedom on the vortex tunneling. Inserting the current $J^{\perp}$ from Eq. (17) into Eq. (12), one gets

$$
S_{e n v}=\frac{\sigma}{2 \beta c^{2}} \int d \tau_{1} d \tau_{2} \sum_{n}\left|\omega_{n}\right| e^{-i \omega_{n}\left(\tau_{1}-\tau_{2}\right)} \int \frac{d^{2} q}{(2 \pi)^{2}} d z e^{i \mathbf{q}\left(\mathbf{R}\left(\tau_{1}\right)-\mathbf{R}\left(\tau_{2}\right)\right)} \frac{-i \mathbf{q} \times \mathbf{A}_{v o r}(-\mathbf{q}, z)}{q} \frac{i \mathbf{q} \times \mathbf{A}_{v o r}(\mathbf{q}, z)}{q} .
$$

Performing the sum over the frequencies and rewriting the action in terms of the magnetic field we get eventually:

$$
S_{e n v}=-\frac{\pi \sigma}{2 \beta^{2} c^{2}} \int d \tau_{1} d \tau_{2} \int \frac{d^{2} q}{(2 \pi)^{2}} d z \frac{e^{i \mathbf{q}\left(\mathbf{R}\left(\tau_{1}\right)-\mathbf{R}\left(\tau_{2}\right)\right)}}{\sin ^{2}\left(\frac{\pi}{\beta}\left(\tau_{1}-\tau_{2}\right)\right)} \frac{B_{v o r}^{z}(-\mathbf{q}, z) B_{v o r}^{z}(\mathbf{q}, z)}{q^{2}}
$$

\section{TUNNELING IN THE PRESENCE OF $S_{e n v}$}

For the purpose of illustration, let us compare $S_{\text {env }}$ with the action of Refs $\stackrel{18,30}{ }$ for a particle moving in a dissipative environment:

$$
S_{C L}=1 / 4 \pi \int d \tau_{1} d \tau_{2} \eta \frac{\left(\mathbf{R}\left(\tau_{1}\right)-\mathbf{R}\left(\tau_{2}\right)\right)^{2}}{\left(\tau_{1}-\tau_{2}\right)^{2}} .
$$

This term results from integrating out the slow degrees of freedom of the environment. The long-time response of these modes reveals itself through a non-local in time term in the action. If $\mathbf{R}(\tau)$ describes a particle moving with a constant velocity, one can substitute $\left[\mathbf{R}(\tau)-\mathbf{R}\left(\tau^{\prime}\right)\right] /\left(\tau-\tau^{\prime}\right)$ with the velocity $\dot{\mathbf{R}}$. Then the action $S_{C L}$ reduces to $1 / 2 \pi \int d \tau_{1} d \tau_{2} \eta \dot{\mathbf{R}}^{2} / 2$ where the integrand is a reminiscent of the Rayleigh's dissipation function. The Rayleigh function is used in the EulerLagrange equations to include dissipation ${ }^{31}$ :

$$
\frac{d}{d t}\left(\frac{\partial \mathcal{L}}{\partial \dot{R}}\right)-\frac{\partial \mathcal{L}}{\partial R}+\frac{\partial \mathcal{F}}{\partial \dot{R}}=0
$$

where $\mathcal{F}=\eta \dot{R}^{2} / 2$ is the Rayleigh function with a friction coefficient $\eta$. Since the Rayleigh function enters the equation of motion without a time derivative (unlike the Lagrangian), the inclusion of the dissipation into the action costs an additional time integration. Therefore, the corresponding term must be non-local in time.
Under the same approximation of constant velocity, the action $S_{e n v}$ can be written in the coordinate representation as:

$$
S_{\text {env }}=\frac{1}{4 \pi} \int d^{2} r d z \int d \tau_{1} d \tau_{2} \frac{\sigma}{c^{2}}\left[\mathbf{v}_{v o r} \times \mathbf{B}_{v o r}(\mathbf{r}, z)\right]^{2} .
$$

The combination $\left[\mathbf{v}_{\text {vor }} \times \mathbf{B}_{\text {vor }}(\mathbf{r})\right] / c$ is the electric field created by a moving vortex. Then, the integrand in the action $S_{e n v}$ is merely the energy dissipation rate in the gate caused by the vortex motion, $S_{\text {env }}=$ $1 / 2 \pi \int d^{2} r d z \int d \tau_{1} d \tau_{2} \sigma E_{\text {vor }}^{2}(\mathbf{r}, z) / 2$. This expression is in full correspondence with the one obtained for a constant motion of a particle in the presence of friction $\eta$.

We return now to Eq. (20) and analyze it for the case of tunneling between two minima separated by a distance $\delta r$. We start with the integration over the coordinate $z$ and momentum $\mathbf{q}$ using for the magnetic field $\mathbf{B}_{\text {vor }}$ the solution given by Eq. (2). We get in result

$$
\begin{aligned}
S_{\text {env }}=\frac{\alpha^{2} \sigma d}{16 e^{2} \lambda^{2}} & \int d \tau_{1} d \tau_{2} \frac{\left(\mathbf{R}\left(\tau_{1}\right)-\mathbf{R}\left(\tau_{2}\right)\right)^{2}}{\left(\tau_{1}-\tau_{2}\right)^{2}} \\
& \times \ln \left(\frac{d}{\lambda}+\frac{8 \pi^{2} \sigma^{2 D} d}{\left|\tau_{1}-\tau_{2}\right| c^{2}}\right)^{-1} .
\end{aligned}
$$

The appearance of the log-factor is very natural if one recalls that we integrate the square of the magnetic field and it is well known that $B_{\text {vor }} \sim 1 / r$ at a distance $r$ 
from the center of the vortex, when $r<\lambda$. The time dependence of the logarithm results from the fact that the integration over the momenta is limited to the Ohmic regime, $\left|\omega_{n}\right| \ll c^{2} q /\left(2 \pi \sigma^{2 D}\right)$. The time dependence of the logarithm is important because in thin superconducting films the ratio $d / \lambda$ can be very small. At large time differences, which are essential for low temperatures, the logarithmic factor in the action becomes $\ln \lambda / d$.

Following the standard Renormalization Group (RG) procedure one gets that the modified tunneling rate $\Gamma_{G}$ is:

$$
\Gamma_{G}(T)=\Gamma_{u n G}\left(T \tau_{\text {tun }}\right)^{K_{E}},
$$

where the exponent $K_{E}$ is equal to the dimensionless dissipation coefficient

$$
K_{E}=\sigma d \frac{(\alpha \delta r)^{2}}{8 e^{2} \lambda^{2}} \ln \left(\frac{\lambda}{d}\right) .
$$

In Eq. (25), $\tau_{\text {tun }}$ is the time of the under-barrier motion of the vortex in the process of tunneling in the absence of the gate (i.e., the duration of the fast stage of the tunneling discussed in the Introduction). The parameter $\tau_{\text {tun }}^{-1}$ acts as the high-energy cutoff because only slow excitations that cannot follow adiabatically the tunneling particle contribute to the slow stage of the tunneling process (it is assumed that $\tau_{\text {tun }}^{-1}>>T$ ). Since only the current in the Ohmic regime contributes to $S_{\text {env }}$, there is an additional high-energy cutoff $\sim c^{2} /\left(4 \pi \sigma^{2 D} d\right)$. Therefore, in Eq. (25) $\tau_{\text {tun }}^{-1}$ should be replaced by $\tilde{\tau}_{\text {tun }}^{-1}=$ $\min \left\{\tau_{\text {tun }}^{-1}, c^{2} /\left(4 \pi \sigma^{2 D} d\right)\right\}$. Little can be said about $\tau_{\text {tun }}$ as the effective mass of the vortex and the potential of the tunneling barrier depend on the specific properties of the superconducting film. As to the energy scale $c^{2} /\left(4 \pi \sigma^{2 D} d\right)$, it is evaluated to be $\sim 10^{3} K$ (the resistivity of the gate is about $10 \mu \Omega \mathrm{cm}$ ).

The temperature enters in Eq. (25) as a low-energy cutoff because the excitations with energy smaller than $T$ do not contribute to the action. The dependence of the tunneling rate on other factors limiting the time of response of the environment can be found from the RG analysis $\frac{16,32}{12}$ Apart from the temperature, such factors include the typical energy mismatch between the minima of the vortex potential $\varepsilon$ and the tunneling rate itself. The energy mismatch $\varepsilon$ in this problem is equivalent to a magnetic field in the Kondo problem. Moreover, lowfrequency components of the Eddy current with frequencies smaller than $\Gamma_{G}$ cannot develop when the tunneling events are too frequent. This is why the tunneling rate determines its own renormalization in a self-consistent way. To include the influence of these factors, one should substitute $T$ in Eq. (25), by $T_{\max }=\max \left\{T, \Gamma_{G}, \varepsilon\right\}$.

For $K_{E}>1$ the temperature dependence given by Eq. (25) holds for all temperatures down to zero. Thus, for $\varepsilon \rightarrow 0$ the vortex becomes localized at $T=0$. In the Kondo problem ${ }^{32}$ this happens for a ferromagnetic sign of the exchange. The localization occurs because the strong response of the environment blocks the tunneling. In the opposite case, $K_{E}<1$, the tunneling rate remains finite when $T, \varepsilon \rightarrow 0$ :

$$
\Gamma_{G} \sim \tilde{\tau}_{\text {tun }}^{-1}\left(\Gamma_{\text {un } G} \tilde{\tau}_{\text {tun }}\right)^{1 /\left(1-K_{E}\right)} .
$$

This quantity has the meaning of the Kondo temperature which determines the physics of switching between two states when both $T$ and $\varepsilon$ are smaller than $\Gamma_{G}$. Unlike the Kondo problem, the dimensionless parameters $K_{E}$ and $\left(\Gamma_{u n G} \tau_{\text {tun }}\right)$ which determine the tunneling rate are completely uncorrelated. We are interested in studying the case when the tunneling is small even in the absence of the gate, i.e., when $\Gamma_{\text {un }} \ll 1 / \tau_{\text {tun }}$. Then, the tunneling between two states is equivalent to the Kondo problem in the limit of extreme anisotropy. In this case the renormalization of $K_{E}$ can be neglected, and the trajectories of the $\mathrm{RG}$ phase-diagram are straight lines.

So far, in the discussion of the renormalization of the tunneling rate we ignored the time dependence of the logfactor in Eq. (24). Generally speaking, this is not valid as there can be a big window of energies, $\tilde{\tau}_{\text {tun }}^{-1}>\left|\omega_{n}\right|>\tau_{\lambda}^{-1}$, where $\tau_{\lambda}^{-1}=c^{2} / 4 \pi \sigma^{2 D} \lambda$. Then, the RG analysis has to be revised. The modified phase diagram is plotted in Fig. 3 see Appendix for details. Notice, that the condition for localization (the inability to tunnel) becomes harder. It is also worth noting that the DelocalizationLocalization phase transition occurs at a finite energy scale.

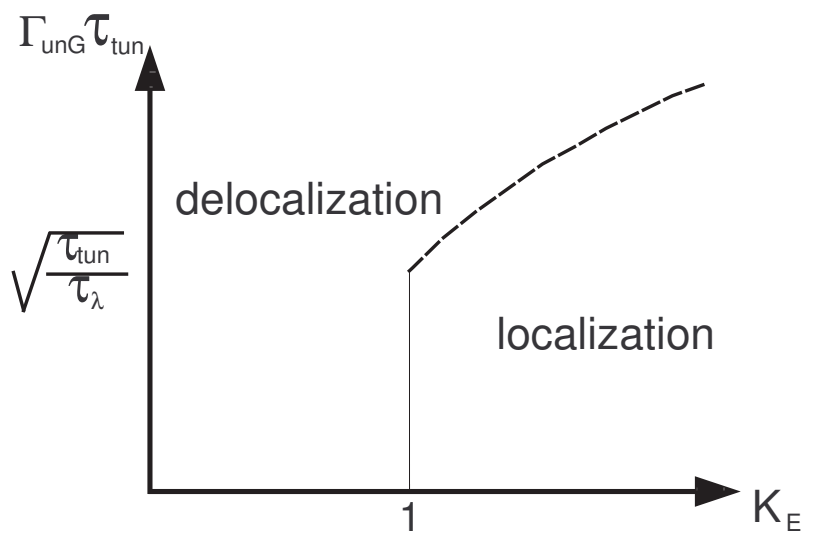

FIG. 3: The Delocalization-Localization phase diagram for the tunneling of vortices in the plane of $\Gamma_{u n G} \tau_{t u n}$ and the dissipation coefficient $K_{E}$.

Let us discuss the result obtained for the exponent $K_{E}$. Naturally, the action $S_{\text {env }}$ is proportional to the square of the magnetic field. After integration over the coordinates one obtains the factor $(\alpha / \lambda)^{2}$. From the structure of the action (24) it follows that $K_{E}$ is proportional to the squared distance of the change of vortex position, which is typically of order $\xi$. Altogether, the exponent $K_{E}$ acquires the factor $(\xi / \lambda)^{2} \ll 1$. This smallness is opposed by the sheet conductance of the gate, $\sigma^{2 D}$, which in the case of a thick well conducting gate gives a large 
factor $2 \pi \hbar \sigma d / e^{2}$. As usually, the effect of dissipation is stronger for cleaner systems.

\section{ORTHOGONALITY CATASTROPHE ${ }^{20}$}

The magnetic flux of a vortex piercing through the gate scatters the electrons in a way similar to Aharonov-Bohm $(A-B)$ scattering $\stackrel{21,22}{=}$ The tunneling of a vortex enforces the electrons inside the gate to adjust to its new position. The response of the electrons to a sudden change of the vortex position leads to the $O C$ that manifests itself in the vanishing overlap $\left\langle\Psi_{f} \mid \Psi_{i}\right\rangle$ of the two wave functions describing the macroscopic electron system before and after the change of the scattering potential 19 The tunneling rate renormalized by the overlap integral is

$$
\Gamma_{G}=\Gamma_{u n G}\left\langle\Psi_{f} \mid \Psi_{i}\right\rangle .
$$

The overlap $\left\langle\Psi_{f} \mid \Psi_{i}\right\rangle$ can be expressed in terms of the operators $\hat{S}_{i}$ and $\hat{S}_{f}$ describing the scattering of the electrons by the magnetic field of the vortex in its initial and final positions 33 :

$$
\begin{aligned}
& \left|\left\langle\Psi_{f} \mid \Psi_{i}\right\rangle\right|=N^{-K_{O C}} \\
& K_{O C}=-\frac{1}{8 \pi^{2}} \operatorname{Tr}\left\{\ln ^{2}\left(\hat{S}_{f} \hat{S}_{i}^{-1}\right)\right\} .
\end{aligned}
$$

Here $N$ is the number of electrons in the gate and, hence, the overlap factor vanishes unless there is a mechanism that limits the effectiveness of the $O C$. It is clear from the comments to Eq. (25) that at finite temperatures $\frac{34,35}{35}$ the parameter $1 / N$ should be substituted by $\left(\max \left\{T, \Gamma_{G}\right\} \tau_{\text {tun }}\right)$. Obviously, the renormalized tunneling rate $\Gamma_{G}$ is given by Eqs. (25) and (27) with $K_{E}$ replaced by $K_{O C}$, and the localization of vortices can be achieved when $K_{O C}>1$.

In the following part of this section we show that for a superconducting film magnetically coupled to a metallic gate (see Fig. 2) the exponent $K_{O C}$ is

$$
K_{O C}=\varsigma d\left(k_{F}^{\text {gate }}\right)^{2} \frac{(\alpha \delta r)^{2}}{64 \lambda},
$$

where $k_{F}^{g a t e}$ is the Fermi momentum of the electrons in the gate and the prefactor $\varsigma$ is evaluated numerically as $\approx 0.4$.

The cylindrical symmetry of the vortex allows us to analyze the scattering of electrons using the basis of cylindrical waves, $\left|\ell, q, k_{z}\right\rangle$; here $\ell$ is the angular momentum along the $z$ axis, while $q$ and $k_{z}$ are the magnitudes of the in-plane and $z$ components of the momentum. In this basis the elements of the matrix $S_{f} S_{i}^{-1}$ can be easily calculated in terms of the phase shifts $\delta_{\ell}$ as

$$
\begin{aligned}
{ }_{f}\left\langle\ell\left|S_{f} S_{i}^{-1}\right| \ell^{\prime}\right\rangle_{f} & \\
& =\sum_{n} e^{2 i \delta_{\ell}-2 i \delta_{n+\ell}} J_{n}(q \delta r) J_{n-\ell^{\prime}+\ell}(q \delta r),
\end{aligned}
$$

where $J_{\nu}(z)$ is the Bessel function.

To proceed further, we need to find the specific phase shifts for scattering by a vortex. An analogy to classical scattering, where the angular momentum is related to the impact parameter $b=|\ell| / q$, helps elucidate the behavior of the phase shift as a function of $\ell$. For $b \gg \lambda$, the scattering by the vortex is similar to the $A-B$ scattering by a flux $\alpha \Phi_{0}$. In the $A-B$ scattering 21,22 electrons acquire the phase $\delta_{\ell}^{A-B}=\frac{\pi}{2}(|\ell|-|\ell-\alpha|)$. The uniqueness of this scattering is in its infinite range: $\delta_{\ell}$ does not vanish when $|\ell| \rightarrow \infty$. For scattering by the vortex, the jump in the $A-B$ phase shifts is smeared out, but the infinite range character of this scattering is preserved. Hence, $\delta_{\ell}$ varies monotonically as a function of $\ell$ between the two limits:

$$
\delta_{\ell} \underset{\ell \gg q \lambda}{\longrightarrow} \alpha \frac{\pi}{2} \operatorname{sgn} \ell .
$$

Naturally, for $q \lambda \gg 1$ the phase shift depends on $b$ and $\lambda$ only through the dimensionless combination $b / \lambda=\ell / q \lambda$ such that $\delta_{\ell}=\frac{\alpha \pi}{2} g(\ell / q \lambda)$; see Fig. 3 in Ref. 20 for illustration.

We now notice that the sum determining the elements of ${ }_{f}\left\langle\ell\left|S_{f} S_{i}^{-1}\right| \ell^{\prime}\right\rangle_{f}$ is accumulated at $-q \delta r \lesssim n \lesssim q \delta r$. This is because the Bessel Functions $J_{\nu}(z)$ decay exponentially with their order when $\nu>z$. Therefore, since $\delta r / \lambda \sim \xi / \lambda \ll 1$, the phase shifts difference in Eq. (31) can be approximated as:

$$
\delta_{\ell}-\delta_{n+\ell} \underset{n \ll q \lambda}{\longrightarrow}-n \delta_{\ell}^{\prime} ; \quad \delta_{\ell}^{\prime} \approx \frac{\alpha \pi}{2 q \lambda} g^{\prime}\left(\frac{\ell}{q \lambda}\right) \ll 1 .
$$

The final step of the calculation is to expand in $\delta r / \lambda$ the logarithm in Eq. (29), and take the trace over $\ell$ and the momentum on the Fermi surface. The outcome of the calculation is given in Eq. (30). The gate thickness $d$ appears here as a result of taking the trace. The specifics of the vortex solution enter only through $g(x)$, with the integral yielding $\varsigma=\int d x(d g / d x)^{2} \approx 0.4$.

Using the known expressions for $\lambda$ and $\xi$ in disordered thin films, the exponent can be rewritten as:

$$
K_{O C} \sim \varsigma \frac{\alpha^{2}}{48 \pi}\left(\frac{e^{2}}{c}\right)^{2} \frac{v_{F}^{s c}}{e^{2}}\left(k_{F}^{\text {gate }} d\right)\left(k_{F}^{\text {gate }} a\right)\left(k_{F}^{s c} l^{s c}\right)^{2} .
$$

The index $s c$ refers to the electrons in the superconducting film: $l^{s c}$ is their mean free path (in the normal state) and $v_{F}^{s c}$ is the Fermi velocity. Interestingly, $T_{c}$ drops out from $K_{O C}$ so that it depends only on the geometrical factors and the non-superconducting properties of electrons. We see that the value of the exponent $K_{O C}$ is determined by a small factor $\sim 10^{-7}$ opposed by a product of a few large factors. Unlike $K_{E}$, the condition for vortex localization can be easily fulfilled by $K_{O C}$ for a not too thin gate and not too disordered superconducting film. 


\section{COMPARISON BETWEEN THE TWO CALCULATIONS AND DISCUSSION}

The expressions in Eqs. (26) and (30) for the exponent describing the renormalization of the tunneling rate have been obtained assuming that only one vortex participates in each tunneling event. In general, vortices can tunnel as a bundle, or as topological defects. In a vortex lattice or in a glass state such defects can be dislocations pairs, interstitials or vacancies. Still, the calculation remains valid as long as $\delta r \ll \lambda$. This is because the magnetic field of the tunneling vortices extends over a large distance, so that their exact configurations before and after the tunneling are not important. The only relevant quantity is the product $\alpha \delta r$. For a single vortex, $\alpha=1 / 2$. When more than one vortex tunnel together $\alpha$ should be multiplied by the number of vortices.

The two expressions for the exponent, $K_{E}$ and $K_{O C}$, share the same dependence on $\alpha \delta r$ and $d$. Therefore, the ratio between them is "universal":

$$
\frac{K_{E}}{K_{O C}}=\frac{\sigma \ln (\lambda / d)}{e^{2}\left(k_{F}^{\text {gate }}\right)^{2} \lambda} \sim \frac{l_{\text {gate }}}{\lambda},
$$

where $l_{\text {gate }}$ is the mean free path of the electrons in the gate. Since in a thin superconducting film the penetration depth $\lambda$ is very large, under the conditions of the experiment ${ }^{\underline{9}}$, the $O C$ is dominant.

In order to understand the difference between the two expressions for the exponent, we have to explain the dependence of $K_{O C}$ on $\lambda$. Although we invoke the expansion in terms of the small parameter $\delta r / \lambda \ll 1$, we get $K_{O C} \propto(\delta r)^{2} / \lambda$. This is typical for the $O C$ when an extended scattering potential is considered, because in this case a large number of scattering channels (harmonics) is involved. Therefore, the total effect of $O C$ is parametri-

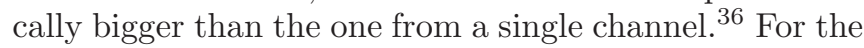
problem discussed here, the relatively weak dependence of $K_{O C}$ on $\lambda$ can be understood from the following arguments. It has been shown that the $O C$ is determined by $\sum_{\ell}\left(\delta_{\ell}-\delta_{\ell+1}\right)^{2} \approx \sum_{\ell}\left(\delta_{\ell}^{\prime}\right)^{2}$. Since the phase shifts approach asymptotically the limit $\pm \alpha \frac{\pi}{2}$, the sum

$$
\sum_{\ell}\left(\delta_{\ell}-\delta_{\ell+1}\right) \approx \sum_{\ell} \delta_{\ell}^{\prime}=\pi \alpha .
$$

Therefore, the result obtained for the exponent $K_{O C}$ corresponds to the differences $\left(\delta_{\ell}-\delta_{\ell+1}\right)$ that are distributed almost equally between $L \sim q \lambda$ channels:

$$
\sum_{\ell}\left(\delta_{\ell}-\delta_{\ell+1}\right)^{2} \sim L\left(\frac{\pi \alpha}{L}\right)^{2} \sim \frac{\alpha^{2}}{\lambda} .
$$

Indeed we see that the first power of $\lambda^{-1}$ is natural for the exponent $K_{O C}$ because a large number of scattering channels is perturbed by the change in the potential when such an extended object as a vortex tunnels.

The peculiarity of the discussed problem is that a vortex in a thin film is a very extended object. In general, the tunneling of an extended object excites many channels of the environment. Unlike the standard OC caused by a scattering potential (but not a vector potential) where the exponent is determined by sum of squares of the phase shifts, here the sum of the squared derivatives of the phase shifts determines the exponent of the OC. Still there is some similarity between the two problems. In the standard OC the sum of the phase shifts is finite because of the Friedel sum rule (see e.g. the tunneling via the localized level considered in Ref. 36), while in the discussed problem the asymptotic limits of the $\mathrm{AB}$ phase shifts make the sum of the derivatives of the phase shifts to be finite, see Eq. (36). As a result of this "AB sum rule" the OC exponent given by Eq. (37) is similar to that given by Eq. (10) in Ref. 36 .

One may conclude from Eqs. (36) and (37) that the randomization of the phase shifts due to the disorder can only increase the value of the exponent $K_{O C}$. (In the general case, $\ell$ should be substituted by the index of the states diagonalizing the scattering matrix.) The scattering by impurities leads to the randomization of the phase differences, while the asymptotic limits of the phase shifts remain the same, $\pm \alpha \frac{\pi}{2}$. Therefore, the value of the exponent $K_{O C}$, which is determined by the squares of the phase differences, should increase in the presence of disorder. This conclusion is in accordance with the existing theoretical results on the enhancement of the $O C$-exponent by not too strong disorder 37,38

The sum over the channels (harmonics) enters in a natural way into the exponent $K_{O C}$, while in the calculation of the "tunneling with dissipation" the sum is absent. The scheme of calculation of the "tunneling with dissipation" for the Eddy currents corresponds to the OC expression calculated in the perturbation theory up to the second order with respect to the change of the vector potential. It is merely finding the elements of the scattering matrix in the Born approximation. However, the $\mathrm{AB}$ effect is a non-perturbative phenomenon. When the current is calculated in the linear response the asymptotic limits of the phase shifts are not captured. One may also see that in the linear response scheme only one channel remains in the sum over the channels that is present in the OC expression. Therefore, the additional factor of $\lambda$ cannot be reproduced within the "tunneling with dissipation" scheme. This is the reason why $K_{O C}$ is significantly larger than the obtained $K_{E}$.

The idea to use a double layer system to study the dynamics of vortices is well known $39,40,41$ In addition to the magnetic coupling between the film and the gate, one may consider a capacitive coupling between them. In the case of the Josephson junction arrays (or granular superconductors) the capacitive coupling reduces the fluctuations of the phase of the superconducting order parameter $\stackrel{41,42}{ }$ As a result, the system may undergo a transition from an insulating to a superconducting state. However, for a homogenous film with a relatively small resistance $\sim 1.5 k \Omega / \square$ used in Ref. 9 the phase fluctuations are not so effective $\underline{\underline{43}}$ This is confirmed by the observed insensitivity of the critical magnetic field $H_{c}$ to the presence of 
the gate. Furthermore, in homogeneous superconductors the motion of vortices is not accompanied by the redistribution of the charge density. Therefore, there are good reasons to ignore here the capacitive coupling between the film and the gate.

\section{SUMMARY}

In this work we have studied how a metallic gate placed above a superconducting film affects the tunneling rate of the vortices. The gate and the film are coupled by the magnetic field of the vortices that pierces through the gate. We analyze the renormalization of the tunneling rate by the gate. We consider two approaches to describe the response of the electrons inside the gate on the tunneling event: (i) the Eddy current in the gate generated by the motion of vortices, and (ii) the OC caused by the change in the vortex position. The OC is due to the Aharonov-Bohm scattering of electrons inside the gate. The exponent determining the renormalized tunneling rate $\Gamma_{G}(T)=\Gamma_{\text {un }}\left(T \tau_{\text {tun }}\right)^{K}$ is given by Eq. (26) for the effect of the Eddy current, and by Eq. (30) for the OC. We find that for the experimental setup of Ref. 9 the effect of the OC provides an exponent sufficient for a substantial suppression of the tunneling rate of the vortices, $K_{O C} \sim 1$.

The peculiarity of the discussed problem results from combination of two elements: the extended size of the tunneling vortex and the unique features of the $\mathrm{AB}$ scattering. In general, the tunneling of an extended object creates excitations in many channels of the environment. The sum over the channels (harmonics) enters in a natural way into the exponent describing the effect of the OC, while in the calculation of the "tunneling with dissipation" the sum is absent. This is the reason why $K_{O C}$ is significantly larger than the obtained $K_{E}$. The scheme of calculation of the "tunneling with dissipation" for the Eddy currents corresponds to the OC expression calculated in the perturbation theory up to the second order with respect to the change of the vector potential. Because of the non-perturbative character of the $\mathrm{AB}$ effect, the phase shifts cannot be found within the Born approximation. Therefore the action $S_{\text {env }}$, being formulated in terms of the macroscopic charge and current densities in the regime of the linear response, is unable to describe the response of all fluctuation modes that can be excited by a vortex as a result of tunneling.

We address our analysis to a recent experiment $\underline{\underline{9}}$ in which the resistance of a superconducting film has been measured in a magnetic field both with and without a gate. We interpret the experiment by assuming that the origin of the resistance at low temperatures is tunneling vortices. From our point of view, the difference in the resistance of the gated and ungated film indicates that the gate reduces the tunneling rate of the vortices making them localized. Indeed, we show here that adding a gate may effectively suppress the vortex tunneling. The gated system discussed here can be used as an effective experimental tool for investigating the vortex motion at low temperatures. The gated system provides a unique opportunity to study the vortex tunneling in thin superconducting films by such simple means as varying the characteristics of the gate, in particular the gate thickness and/or the sheet conductance of the gate. This may help to identify the different mechanisms that contribute to the suppression of the vortex tunneling rate.

\section{Acknowledgments}

We thank A. Kapitulnik, T. M. Klapwijk, B. I. Halperin, D. E. Khmelnitskii, A. D. Mirlin and B. Spivak for useful discussions. AF is supported by the Minerva Foundation.

\section{APPENDIX A: LOCALIZATION-DELOCALIZATION PHASE DIAGRAM FOR EQ. (24)}

The renormalized tunneling rate as given by Eq. (25) corresponds to the RG equation

$$
d \ln \left(\Gamma_{G} \tau\right) / d \ln \left(\tau / \tilde{\tau}_{\text {tun }}\right)=\left(1-K_{E}\right) .
$$

This equation is valid as long as the logarithmic factor in Eq. (24) is independent of the time difference $\left|\tau_{1}-\tau_{2}\right|$. The time dependence in the logarithmic factor is essential for the energy interval $\tilde{\tau}_{\text {tun }}^{-1}>1 / \tau>\tau_{\lambda}^{-1}$, where $\tau_{\lambda}^{-1}=c^{2} / 4 \pi \sigma d \lambda$. Then, the RG equation has to be modified by replacing the dissipation coefficient $K_{E}$ in the above equation by the energy dependent parameter $\tilde{K}_{E}=K_{E}\left[1-\ln \left(\tau_{\lambda} / \tilde{\tau}_{\text {tun }}\right) / \ln (\lambda / d)+\varrho / \ln (\lambda / d)\right]$, with the logarithmic variable $\varrho=\ln \left(\tau / \tilde{\tau}_{\text {tun }}\right)$. For the discussed energy interval the "RG equation" becomes

$$
d \ln \left(\Gamma_{G} \tau\right) / d \varrho=\left(1-\tilde{K}_{E}(\varrho)\right)
$$

One should not be confused with the appearance of $\varrho$ in the RHS of the "RG equation". Here we just integrate $S_{\text {env }}$ in the exponent determining the renormalized tunneling rate $\Gamma_{G} \propto \exp \left(-S_{\text {env }}\right)$. In a sense we calculate something like the Debye-Waller factor created by the dissipative environment $\stackrel{44}{ }$. [The $\varrho$-dependence of the parameter $\tilde{K}_{E}$ can be reformulated as an RG equation, additional to Eq. (A2). Namely,

$$
d \tilde{K}_{E} / d \varrho=\left\{\begin{array}{cl}
\text { const } & 0<\varrho<\ln \left(\tau_{\lambda} / \tilde{\tau}_{\text {tun }}\right) \\
0 & \varrho>\ln \left(\tau_{\lambda} / \tilde{\tau}_{\text {tun }}\right)
\end{array}\right.
$$

where const $=K_{E} / \ln (\lambda / d)$. Because of the different dependencies of $\tilde{K}_{E}$ on $\varrho$, the RG process may develop in two steps. We discuss the details below.]

Localization occurs if in the course of the RG process the tunneling rate $\Gamma_{G}$ decreases faster than the running scale $\tau^{-1}$. Hence, the line separating the localized 
and delocalized states is determined by the condition $\tilde{K}_{E}\left(\Gamma_{G}\right)=1$ as long as $\Gamma_{G}>\tau_{\lambda}^{-1}$. Since the expression for $\tilde{K}_{E}$ assumes that $\varrho<\ln \left(\tau_{\lambda} / \tilde{\tau}_{\text {tun }}\right)$, the modified $\tilde{K}_{E}$ is smaller than $K_{E}$. Therefore, the condition for localization (the inability to tunnel) becomes harder. It is worth noting that in the discussed problem the phase transition occurs at a finite energy scale

$$
\ln \left(1 / \Gamma_{G} \tilde{\tau}_{\text {tun }}\right)=\frac{\ln (\lambda / d)}{K_{E}}-\left[\ln (\lambda / d)-\ln \left(\tau_{\lambda} / \tilde{\tau}_{\text {tun }}\right)\right]
$$

To avoid unnecessary complications, from now on we limit ourselves to the case when the energy cutoff $1 / \tilde{\tau}_{\text {tun }}$ is determined by $c^{2} / 4 \pi \sigma d^{2}$. Then $\ln \left(\tau_{\lambda} / \tilde{\tau}_{\text {tun }}\right)=\ln (\lambda / d)$ and Eq. A3 reduces to $\ln \left(1 / \Gamma_{G} \tilde{\tau}_{\text {tun }}\right)=\ln \left(\tau_{\lambda} / \tilde{\tau}_{\text {tun }}\right) / K_{E}$. Note that the line of the phase transition exists for all $K_{E}>1$ and in the delocalized phase $\Gamma_{G}>\tau_{\lambda}^{-1}$. Clearly for $K_{E}<1$ the tunneling rate always remains finite.

In order to find the line of the DelocalizationLocalization transition in the plane of the dimensionless parameters $\left(\Gamma_{u n G} \tilde{\tau}_{\text {tun }}\right)$ and $K_{E}$, one has to integrate back Eq. (A2) starting at $\ln \left(\Gamma_{G} \tau\right)=0$. (The value of $\Gamma_{G}$ corresponding to the line of transition should be found from the condition $\left.\tilde{K}_{E}\left(\Gamma_{G}\right)=1\right)$. This procedure yields for the boundary between the two phases

$$
\ln \left(1 / \Gamma_{\text {unG }} \tilde{\tau}_{\text {tun }}\right)=\ln \left(\tau_{\lambda} / \tilde{\tau}_{\text {tun }}\right) / 2 K_{E}
$$

One may see that along the boundary $\left(\Gamma_{G} \tilde{\tau}_{\text {tun }}\right)=$ $\left(\Gamma_{\text {unG }} \tilde{\tau}_{\text {tun }}\right)^{2}$.

In the delocalized phase, the renormalized tunneling rate can be found by integrating the $\mathrm{RG}$ equation starting from the bare tunneling rate down to the energy when $\ln \left(\Gamma_{G} \tau\right)=0$. Since for $K_{E}>1$ the condition $\ln \left(\Gamma_{G} \tau\right)=$ 0 is satisfied before the running scale $\tau^{-1}$ reaches $\tau_{\lambda}^{-1}$, the RG process involves only Eq. (A2). For $K_{E}<1$ the situation is more delicate. In the first step of the RG process Eq. A2 is integrated. If $\tau^{-1}$ reaches $\tau_{\lambda}^{-1}$ before $\ln \left(\Gamma_{G} \tau\right)=0$ (i.e., $\Gamma_{G}<\tau_{\lambda}^{-1}$ ), the process should be continued. (In terms of the bare parameters, this occurs when $\left.\Gamma_{\text {un } G}<\tau_{\lambda}^{-1}\left(\tau_{\lambda} / \tilde{\tau}_{\text {tun }}\right)^{K_{E} / 2}\right)$. In the second step one has to integrate Eq. A1 using $\tau_{\lambda}^{-1}$ as an upper cutoff instead of $\tilde{\tau}_{\text {tun }}^{-1}$ and $\Gamma_{G}\left(\tau_{\lambda}^{-1}\right) \equiv \Gamma_{\lambda}$ as an initial value; here $\Gamma_{\lambda}=\Gamma_{u n G}\left(\tilde{\tau}_{\text {tun }} / \tau_{\lambda}\right)^{K_{E} / 2}$ is the result of the integration in the previous step for $\Gamma_{G}$. Finally, after the two-steps renormalizion the tunneling rate is

$$
\Gamma_{G} \sim \tau_{\lambda}^{-1}\left(\Gamma_{\lambda} \tau_{\lambda}\right)^{1 /\left(1-K_{E}\right)}
$$

which is equivalent to

$$
\Gamma_{G} \tilde{\tau}_{\text {tun }} \sim\left(\Gamma_{\text {un } G} \tau_{\text {tun }}\right)^{1 /\left(1-K_{E}\right)}\left(\tilde{\tau}_{\lambda} / \tilde{\tau}_{\text {tun }}\right)^{K_{E} / 2\left(1-K_{E}\right)}
$$

The Delocalization-Localization phase diagram for the tunneling of vortices in the plane of $\Gamma_{u n G} \tau_{\text {tun }}$ and the dissipation coefficient $K_{E}$ at zero temperature is presented in Fig. 3,

Finally, we touch upon the role of the relativistic effects. In the derivation of the effective action (24) the integration over the momenta has been limited to the Ohmic regime, $q \gtrsim 2 \pi \sigma^{2 D}\left|\omega_{n}\right| / c^{2}$. We are interested in a gate with high conductivity, such that $2 \pi \sigma^{2 D} / c \gg 1$. In this case there are relativistic effects that have been left out from the action. As it has been mentioned in Sec. III, at high frequencies $\left|\omega_{n}\right| / c>q$ the dissipation occurs through the Cherenkov radiation. The produced dissipation is equivalent to an effective two-dimensional conductivity that is approximately $c / 2 \pi$. Therefore, this source of the dissipation is negligible compared to the Ohmic dissipation and cannot change our conclusions.
1 J. Bardeen, and M. J. Stephen, Phys. Rev. 140, 1197 (1965).

2 A. I. Larkin, Zh. Eksp. Teor. Fiz. 58, 1466 (1970) [Sov. Phys. JETP. 31, 784 (1970)].

3 A. I. Larkin, and Yu. N. Ovchinnikov, Zh. Eksp. Teor. Fiz. 61, 1221 (1971) [Sov. Phys. JETP. 34, 651 (1972)].

4 P. W. Anderson, and Y. B. Kim, Rev. Mod. Phys. 36, 39 (1964).

5 Y. Liu, D. B. Haviland, L. I. Glazman, and A. M. Goldman Phys. Rev. Lett. 68, 2224 (1992).

${ }^{6}$ D. Ephron, A. Yazdani, A. Kapitulnik, and M. R. Beasley, Phys. Rev. Lett. 76, 1529 (1996).

7 A. van Oudenaarden, S. J. K. Vardy, and J. E. Mooij, Phys. Rev. Lett. 77, 4257 (1996).

8 F. Tafuri, J. R. Kirtley, D. Born, D. Stornaiuolo, P. G. Medaglia, P. Orgiani, G. Balestrino, and V. G. Kogan, Europhys. Lett. 73, 948 (2006).

9 N. Mason, and A. Kapitulnik, Phys. Rev. B. 65, 220505(R) (2002).
10 The quantum tunneling of vortices leading to the variablerange hopping resistivity in superconducting films has been discussed by M. P. A. Fisher, T. A. Tokuyasu, and A. P. Young, Phys. Rev. Lett. 66, 2931 (1991).

11 N. F. Mott, Metal Insulator Transitions (Taylor and Francis, London, 1974).

12 S. V. Iordanskii, and A. M. Finkel'stein, J. Low. Temp. Phys. 10, 423 (1973).

13 In Ref. 12 the slow stage precedes the fast one preparing the environment for the transition of the fast degress of freedom. In fact, it can happen in the inverse order.

14 In the case of the quatntum nucleation, the slow stage yields a stronger dependence of the action on the size of the nuclea when it is large.

15 A. J. Leggett, S. Chakravarty, A. T. Dorsey, M. P. A. Fisher, A. Garg, and W. Zwerger, Rev. Mod. Phys. 59, 1 (1987) and references therein.

16 A. Schmid, Phys. Rev. Lett. 51, 1506 (1983); the correct form of the phase diagram is given by S. A. Bulgadaev, Pis'ma Zh. Eksp. Teor. Fiz. 39, 264 (1984) [Sov. Phys.- 
JETP Lett. 39, 315 (1984)].

17 C. P. Herrero, and A. D. Zaikin, Phys. Rev. B. 65, 104516 (2002);

18 A. O. Caldeira, and A. J. Leggett, Phys. Rev. Lett. 46, 211 (1981).

19 P. W. Anderson, Phys. Rev. Lett. 18, 1049 (1967)

${ }^{20} \mathrm{We}$ have already discussed the OC caused by the Aharonov-Bohm effect in K. Michaeli, and A. M. Finkel'stein, Phys. Rev. Lett. 97, 117004 (2006); here it is included to make the paper self contained.

21 Y. Aharonov, and D. Bohm Phys. Rev. 115, 485 (1959).

${ }^{22}$ Y. Aharonov, C. K. Au, E. C. Lerner, and J. Q. Liang, Phys. Rev. D 29, 2396 (1984).

23 J. Pearl, Appl. Phys. Lett. 5, 65 (1964).

24 A. A. Abrikosov, Fundamentals of the Theory of Metals (Elsevier, Amsterdam, 1988).

25 G. Carneiro, and E. H. Brandt, Phys. Rev. B 61, 6370 (2000).

26 B. L. Altshuler, A. G. Aronov, and D. E. Khmel'nitskii, J. Phys. C. 15, 7367 (1982)

27 L. S. Levitov, and A. V. Shytov, Pis'ma Zh. Eksp. Teor. Fiz. 66, 200 (1997) [Sov. Phys.JETP Lett. 66, 214 (1997)].

28 V. N. Popov, Functional Integrals in Quantum Field Theory and Statistical Physics (Reidel, Dordrecht, 1983).

29 V. I. Fal'ko, and D. E. Khmel'nitskii, Zh. Eksp. Teor. Fiz. 95, 1988 (1989) [Sov. Phys. JETP. 68, 1150 (1989)].

30 A. O. Caldeira, and A. J. Leggett, Ann. Phys. (NY) 149,
$374(1983)$

31 H. Goldstein, Classical Mechanics (Addison-Wesly, Massachusetts, 1980), page 24 .

32 P. W. Anderson, G. Yuval, and D. R. Hamann, Phys. Rev. B. 1, 4464 (1970).

${ }^{33}$ K. Yamada, and K. Yosida Prog. Theor. Phys. 68,1504 (1982).

34 K. Yamada, Prog. Theor. Phys. 72, 195-201 (1984).

35 Yu. Kagan, and N. V. Prokof'ev, Zh. Eksp. Teor. Fiz. 90, 2176 (1986) [Sov. Phys. JETP 63, 1276 (1986)].

36 K. A. Matveev, and A. I. Larkin, Phys. Rev. B 46, 15337 (1992).

37 Y. Chen, and J. Kroha, Phys. Rev. B. 46, 1332 (1992).

38 Y. Gefen, R. Berkovits, I. V. Lerner and B. L. Altshuler, Phys. Rev. B. 65, 081106(R) (2002).

39 I. Giaever, Phys. Rev. Lett. 15, 825 (1964).

40 G. H. Kruithof, P. C. van Son, and T. M. Klapwijk, Phys. Rev. Lett. 67, 2725 (1991).

41 A. J. Rimberg, T. R. Ho, C. Kurdak, J. Clarke, K. L. Campman, and A. C. Gossard, Phys. Rev. Lett. 78, 2632 (1997).

${ }^{42}$ K. H. Wagenblast, A. van Otterlo, G. Schon, and G. T. Zimanyi, Phys. Rev. Lett. 79, 2730 (1997).

43 T. V. Ramakrishnan, Physica Scripta. 27, 24 (1989).

44 A similar procedure has been applied for the calculation of the zero bias anomaly in disordered conductors by A. M. Finkel'stein, Zh. Eksp. Teor. Fiz. 84, 168 (1983) [Sov. Phys. JETP. 57, 97 (1983)]. 